\title{
Hospital Information Systems: The Past, Present, and Future
}

\section{Eisuke Hanada}

Department of Information Science, Saga University Graduate School of Science and Engineering, Saga, 840-8502, Japan

\section{Abstract}

Hospital Information Systems (HIS) have been used for more than 40 years. At first they were simple computer applications, but they have developed into complex networks that house storage and and reference systems for clinical information and that provide a support system for the multifaceted staff of modern hospitals. Herein are some comments on the past, present, and future of HIS.

\section{Publication History:}

Received: November 19, 2016

Accepted: May 16, 2017

Published: May 18, 2017

\section{Keywords:}

Hospital information system, Electronic patient record, User interface, Information security

\section{Introduction}

My research focuses are on speech/data communication, electromagnetic compatibility in hospitals, and telemedicine, and I have been responsible for maintaining a university hospital information system (HIS) and communication network. Many modern Japanese hospitals have introduced wireless LAN, which has resulted in improved labor efficiency while providing increased patient safety. However, there are difficulties when introducing wireless LAN such as insuring the safety of medical devices in this era in which ever increasing speed is required while maintaining the necessary range [1].

\section{History and Future of HIS}

In early stages of HIS in Japan, the purpose was to do health care accounting, which was difficult because of the complicated government rules. More recently, HIS has been united with networks, which has enabled doctors to communicate their needs from anywhere in the hospital with a subsystem called an "order-entry system". HIS have been connected to the automated electronic examination systems used in hospitals, which has enabled doctors to access the results of examinations that they processed through the HIS. Because Japanese doctors have historically been legally obliged to keep medical records on paper, the introduction of HIS functions grew slowly. Fortunately, the use of electronic medical records was allowed with the coming of the $21^{\text {st }}$ century. This resulted in the rapid spread of electronic medical records, which is now a basic HIS subsystem. Furthermore, HIS currently includes access to electronic patient records by nurses and other professional staff and supports the records of electronic medication administration by pharmacists. Aside from these, HIS subsystems that allow access to information related to hospital management and that manage accounts that are in arrears are also available.

The above have enabled quick and precise information sharing by the staff of Japanese hospitals through the digitalization of clinical information and improvements in the hospital network infrastructure. Through information sharing, improved coordination has become possible among regional hospitals. In Finland, information sharing is done among all hospitals throughout the country. HIS, which was initially a simple computer, has become a complex system for the accumulation and referencing of clinical information and has developed into an indispensable clinical support system.
From the viewpoint of the HIS architecture, it is now in the process of changing stages from a server/client type to a virtual system. Current HIS networks are employing new technologies, such as wireless LAN and VLAN, and I believe that in the near future HIS will commonly use sensor networks and VoIP technology.

\section{Problems with HIS}

Although the development of HIS has provided numerous benefits, there are many unsolved problems, notably those related to information security and user interface. Providing security is a constant problem for system managers. As a result of enabling access to patient information through networks, even from outside the hospital in many cases, threats such as information leaks and illegal access have increased. Security problems can be divided into those that affect the HIS itself and those that affect the network. Technologies for authentication, restriction of the data flow range, and encryption are under development, but they must be made easier to use.

The interface required by users varies; there is nothing that satisfies all users. The customizable range of the display used in present commercial HIS is small. This is a main factor in user dissatisfaction with HIS. The realization of more easily comprehensible displays and ease of operation are important factors if HIS is to obtain complete acceptance by the medical staff. A comprehensible display must enable "at-a-glance" character recognition and have variable character size. Busy doctors tell me it is important that two or fewer clicks be required for one operation. Various HIS terminal hardware is currently available, including desktop PC's, tablets, and smart phones. The future will see increasing demand for wearable terminals. The amount of information that can be displayed differs with the kind of terminal, thus suitable screens need to be displayed and various input methods must be made available. In almost all cases, the users have to make the necessary adaptation when the environment changes.

"Corresponding Author: Prof. Eisuke Hanada, Department of Information Science, Saga University, Graduate School of Science and Engineering, Saga, 840-8502, Japan; E-mail: hanada@cc.saga-u.ac.jp

Citation: Handa E (2017) Hospital Information Systems: The Past, Present, and Future. Int J Comput Softw Eng 2: 114. doi: https://doi.org/10.15344/2456$4451 / 2017 / 114$

Copyright: (c) 2017 Handa. This is an open-access article distributed under the terms of the Creative Commons Attribution License, which permits unrestricted use, distribution, and reproduction in any medium, provided the original author and source are credited. 
Speech recognition is a possible solution to this problem, but though it may be effective in some environments there are many environments in which I fear the possibility of information leaks.

\section{Conclusion}

In addition to the above, there are movements toward information extraction using data mining and toward diagnostic assistance using artificial intelligence. Current medical practice is based on the concept of humans curing humans. HIS will not replace doctors and nurses; however, it will grow into a system that supports the hospital staff in a wide variety of situations. I am looking forward to the evolution and expansion of the functions, applications, and ways of interfacing HIS.

\section{Competing Interests}

The authors declare that they have no competing interests.

\section{References}

1. Hanada E, Kudou T, Tsumoto S (2013) Installation of Secure, Always Available Wireless LAN Systems as a Component of the Hospital Communication Infrastructure. J Med Syst 37: 9939. 